\title{
Evolving Quorum Sensing in Digital Organisms
}

\author{
Benjamin E. Beckmann and Phillip K. McKinley \\ Department of Computer Science and Engineering \\ 3115 Engineering Building \\ Michigan State University \\ East Lansing, Michigan 48824 \\ \{beckma24,mckinley\}@cse.msu.edu
}

\begin{abstract}
For centuries it was thought that bacteria live asocial lives. However, recent discoveries show many species of bacteria communicate in order to perform tasks previously thought to be limited to multicellular organisms. Central to this capability is quorum sensing, whereby organisms detect cell density and use this information to trigger group behaviors. Quorum sensing is used by bacteria in the formation of biofilms, secretion of digestive enzymes and, in the case of pathogenic bacteria, release of toxins or other virulence factors. Indeed, methods to disrupt quorum sensing are currently being investigated as possible treatments for numerous diseases, including cystic fibrosis, epidemic cholera, and methicillin-resistant Staphylococcus aureus. In this paper we demonstrate the evolution of a quorum sensing behavior in populations of digital organisms. Specifically, we show that digital organisms are capable of evolving a strategy to collectively suppress self-replication, when the population density reaches a specific, evolved threshold. We present the evolved genome of an organism exhibiting this behavior and analyze the collective operation of this "algorithm." Finally, through a set of experiments we demonstrate that the behavior scales to populations up to 400 times larger than those in which the behavior evolved.
\end{abstract}

\section{Categories and Subject Descriptors}

I.2.8 [Computing Methodologies]: Artificial IntelligenceProblem Solving, Control Methods, and Search

\section{General Terms}

Experimentation

\section{Keywords}

Artificial life, digital evolution, quorum sensing, multi-agent system, cooperative behavior, self-organization.

Permission to make digital or hard copies of all or part of this work for personal or classroom use is granted without fee provided that copies are not made or distributed for profit or commercial advantage and that copies bear this notice and the full citation on the first page. To copy otherwise, to republish, to post on servers or to redistribute to lists, requires prior specific permission and/or a fee.

GECCO'09, July 8-12, 2009, Montréal Québec, Canada.

Copyright 2009 ACM 978-1-60558-325-9/09/07 ...\$5.00.

\section{INTRODUCTION}

The human body is made up of $10^{13}$ human cells. Yet, this number is an order of magnitude smaller than the number a bacterial cells living within the gastrointestinal tract of a single adult [4]. Although it was previously assumed that bacteria and other microorganisms rarely interact [24], in 1979 Nealson and Hastings found evidence that bacterial communities of Vibrio fischeri and Vibrio harveyi were able to perform a coordinated behavioral change, namely emitting light, when the cell density rose above a certain threshold [20]. This type of density-based behavioral change is called quorum sensing [27]. In quorum sensing (QS), continual secretion and detection of chemicals provide a way for bacteria to assess local cell density. Reaching a sufficient density can trigger expression of genes that produce behaviors more likely to succeed under such conditions.

The initial discovery of Nealson and Hastings spawned a new branch of research to discover the nature of such interactions, whether they occur in other microorganisms, and the consequences of these behaviors. QS has since been observed in many species of bacteria, which use it for a variety of purposes, including secretion of digestive enzymes in the gastrointestinal tract [6], bioluminescence and phototrophy in marine bacteria $[3,20]$, and, in the case of pathogenic bacteria such as Salmonella and Staphylococcus, release of toxins or other virulence factors $[7,9,18]$. QS is also known to be closely related to more complex behaviors, such as aggregation into biofilms [12] and even fruiting bodies [10]. For example, when confronted with starvation due to nutrient depletion, Myxococcus xanthus bacteria cooperate to form a stalk, enabling some cells to be carried as spores to new locations where conditions might be better.

Improved understanding of QS has numerous scientific benefits [7]. Foremost, diseases caused by quorum-sensing bacteria might be treated with medications that inhibit this behavior (i.e., quorum quenching) [18], an approach that may have milder side effects than some antibiotics. For example, Davies et al. [8] showed that QS is essential to the development of biofilms in Pseudomonas aeruginosa, the primary pathogen observed in the lungs of people with cystic fibrosis. In addition, quorum quenching has been proposed as a possible treatment for methicillin-resistant Staphylococcus aureus [23], some strains of which are resistant to most traditional antibiotics [16]. Moreover, a deeper understanding of these interactions and their evolution may provide insight into the evolution of multicellularity itself. 
In addition to furthering biological studies, knowledge of how relatively simple organisms cooperate to perform complex tasks may also be beneficial to the development of distributed computational systems that need to tolerate dynamic conditions and survive component failures as well as cyber-attacks. For example, collective behaviors among agents in an artificial immune system are essential to detecting and responding to potential threats, while nodes in sensor networks need to implement complex distributed operations such as multicasting, gathering sensed data, and maintaining a network topology. Many traditional algorithms for solving these problems are brittle when deployed in dynamic environments, and several promising algorithms proposed recently are inspired by biology [2]. Leveraging the evolutionary process to produce well adapted systems is a logical next step.

In this paper we demonstrate the evolution of QS behavior in populations of self-replicating digital organisms. Specifically, we show that digital organisms in the Avida system [22] are capable of evolving a strategy to collectively suppress self-replication when the population density reaches an evolved threshold. We describe the operation of an evolved genome exhibiting this behavior and analyze the collective operation of a population of such organisms. We also show that the behavior scales to populations up to 400 times larger than those in which the behavior evolved. This study represents a first step in using artificial life, specifically digital organisms, to investigate the evolution and operation of QS.

\section{BACKGROUND}

\subsection{Quorum Sensing in Bacteria}

Bacteria that participate in QS continuously release signaling molecules, called autoinducers (AIs) [21], which they can also detect with AI receptors. Under low-density conditions, AI molecules diffuse throughout the environment and go undetected by the bacteria. However, the level of AI increases directly with cell density and, if it exceeds a threshold, the detection mechanism in the bacteria causes the up regulation of the genes that produce AI molecules. This creates a positive feedback loop which greatly increases the level of AI in the environment. Once a receptor has been fully activated by a high concentration of AI, the activated receptor causes the up or down regulation of other genes in the bacteria. If the level of AI is relatively uniform throughout the environment, all of the bacteria that respond to the high level of AI will begin transcription of the same genes at approximately the same time, thereby changing the population's behavior once a quorum has been reached.

In addition to numerous wet lab studies of QS and biofilm formation [6-8,18,20,27], several researchers have constructed mathematical models that describe gene expression in QS bacteria $[5,25,26]$. These works differ from ours in that they use $\mathrm{P}$ systems to model known gene expression mechanisms. In addition, Nadell et al. [19] recently simulated pairwise evolutionary competitions to investigate the production of extracellular polymeric substances (EPS) used in biofilm formation. To our knowledge, however, the study described in the remainder of this paper is the first to demonstrate the evolution of QS behavior in self-replicating digital organisms.

\subsection{Avida Overview}

Avida is a well established artificial life platform used in evolutionary biology $[1,14,15,22]$ and more recently in distributed systems research [17]. In Avida, individuals, or digital organisms, compete for space within a fixed-size twodimensional collection of cells. Each cell can contain at most one organism, which comprises a circular list of instructions (its genome) and a virtual CPU that executes those instructions, as shown at the top of Figure 1. Instructions perform simple arithmetic operations (addition, bit-shift, increment, etc.), control execution flow, aid in self-replication, and provide a means for the organism to interact with other organisms and the environment. The execution of an instruction costs both virtual CPU cycles and energy. Different instructions can be assigned different CPU cycle and energy costs. An organism executes instructions on its virtual CPU, which contains three general purpose registers (AX, BX, CX), two general purpose stacks, and special purpose heads that point to locations within an organism's genome. Similar to a traditional program counter and stack pointer, heads are used to control the flow of execution.

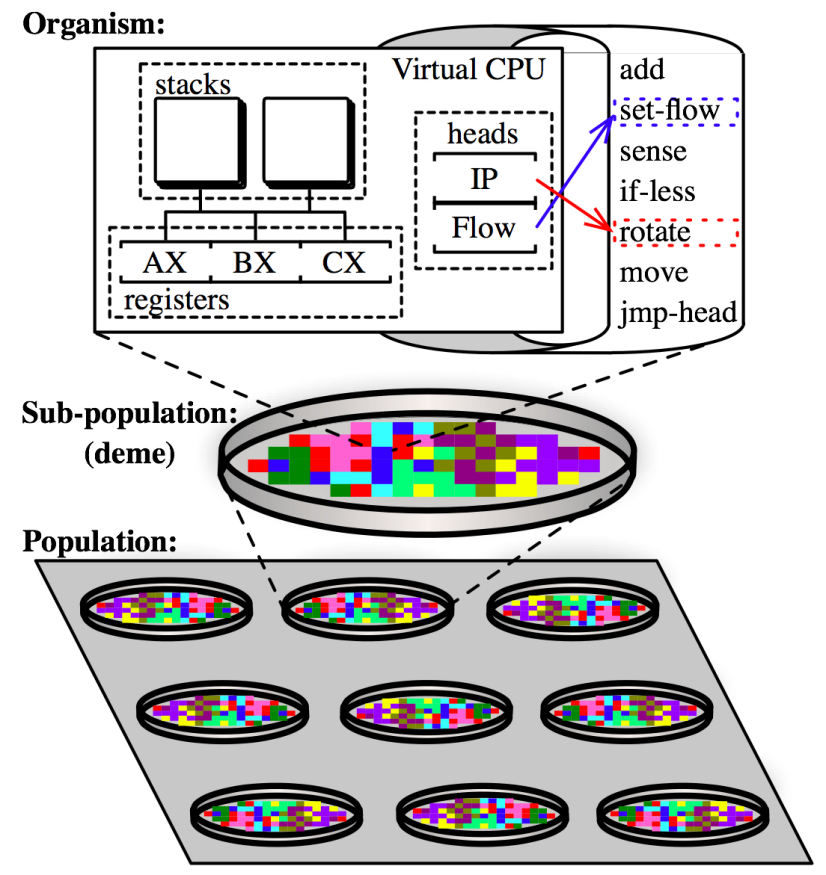

Figure 1: Population (bottom), sub-population (middle) and composition of a digital organism: genome (top right), virtual CPU (top left) with heads pointing to locations within the genome.

Avida organisms are self replicating, that is, their genomes must contain instructions to create offspring. An offspring is placed in a randomly selected cell, terminating any previous inhabitant. Typically, an Avida population starts with a single ancestral seed organism capable only of replication. As organisms replicate, instruction-level mutations produce variation within the population. In this study, the ancestral organism contains 49 no-operation (nop) instructions and a single repro instruction, which performs self-replication. The nop instructions have no effect on the ancestral organism's observed behavior, or phenotype, excluding its gesta- 
tion time. However, they do provide the evolutionary process more room to work, as mutations during replication bring different instructions into the genome with selective pressures, discussed below, favoring resulting behaviors that benefit the organism and its offspring.

Each organism has an energy store that is used to calculate its metabolic rate using Equation 1. An organism's metabolic rate is inversely proportional to a user defined limit on the total number of instructions an organism can execute before its energy is depleted, assuming no new energy influx and all instructions cost 1 energy unit. Probabilistically, an organism with a higher metabolic rate will be given more virtual CPU cycles to execute instructions at a higher energy cost per instruction, calculated by Equation 2 , than an organism with a lower metabolic rate. Generally, an organism can increase its energy, either individually or as part of a group, by exhibiting behaviors prescribed by the user.

$$
\begin{aligned}
& \text { metabolic rate }=\frac{\text { stored energy }}{\text { max. executed inst. }} \\
& \text { actual energy cost }= \text { metabolic rate } \times \\
& \text { inst. } \text { energy cost }
\end{aligned}
$$

In Avida, time is measured using an abstract unit called an update. During an update, an average organism is will probabilistically execute 50 instructions. When exploring a particular problem, we execute several batches of Avida runs, each with a different configuration, and analyze the evolutionary process and resulting behaviors. A batch typically contains of 20 runs, each of which starts with the same ancestral organism but a different random number seed, causing the populations take different evolutionary paths.

\subsection{Demes and Group-level Selection}

In some Avida studies all organisms are treated as part of a single population, in which case individual organisms compete against each other. However, in other studies, especially those involving the evolution of cooperative behavior, it is useful to subdivide a population and have groups of organisms compete. As shown at the bottom of Figure 1, a population of organisms can be subdivided into multiple sub-populations, called demes. While all demes have identical environments and initial configurations, an organism can interact only with other organisms in its deme. Subdividing the population this way is akin to the island model [28] commonly used in evolutionary computation, enabling the detection and eventual selection of demes that perform grouplevel behaviors.

Multilevel selection [29] can be described as the application of natural selection at different granularities. Avida supports multilevel selection, specifically at the individual and deme levels. However, in the work presented here fitness proportional selection is performed only at the demelevel. A deme's fitness is evaluated using Equation 3. After selection and prior to creating an offspring deme, mutations are applied to the genome that was used to seed the parent deme. During this mutation process each instruction in the genome is subject to a $0.75 \%$ chance of being mutated to a random instruction. In addition, there is a $5 \%$ change that a random instruction is inserted and deleted from a random location in the genome. The newly created genome is then used to seed the offspring deme. The sequence of an- cestral genomes make up the germline from which all seed organisms are produced. In contrast, other non-germline, or somatic, organisms play no role in deme replication.

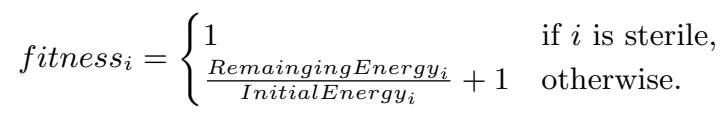

In this work individual organisms within a deme are able to replicate, however those self-replications do not involve mutations to the genome. Hence, all organisms within a deme are genetically identical. Floreano et al. [11] have previously shown that this approach is effective in evolving cooperative behavior.

\subsection{Avida Messaging and Interrupt Handling}

Avida organisms can communicate by sending messages to one another. An Avida message consists of a single packet containing the values of two of the sending organism's registers. The send-msg instruction delivers a message to the organism residing in the currently faced cell. If the cell is unoccupied, then the message fails to be received. An organism can change its facing by executing one of several rotate instructions, discussed later.

In most prior studies using Avida messages, a receiving organism must explicitly retrieve the message from its input buffer in order to process it. However, we recently extended Avida with an interrupt model similar to the execution model of TinyOS [13], an operating system for sensor networks. In this model, depicted in Figure 2, an organism's main execution thread can be interrupted by a particular event, such as receiving a message. To enable context switching of this type, we introduced two instructions that denote the beginning (msg-handler) and end (end-handler) of an interrupt handler. We emphasize that these instructions have simply been added to the set of instructions available for mutation into an organism's genome. Whether they are used or not is solely a result of natural selection.

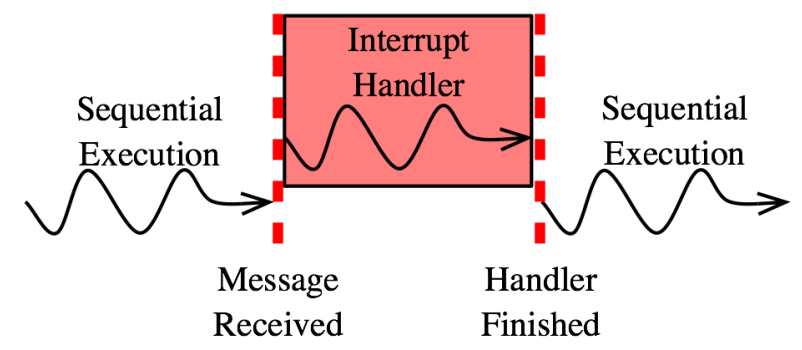

Figure 2: Context switch from sequential execution to an interrupt handler and back.

Figure 3 depicts the semantics of these instructions if they do enter the genome. When an organism receives a message, it genome is searched in the forward direction for the nearest instance of a msg-handler instruction. If none is found, the message is ignored. If a msg-handler instruction does exist, then the organism's context is saved, the contents of the message are placed in two of the organism's registers, and the instruction pointer is moved one instruction past the msg-handler instruction. The interrupt handler returns when an end-handler instruction is executed, which causes the interrupt context to be flushed from the organism's virtual $\mathrm{CPU}$ and the original context to be restored. If no end-handler instruction exists, then execution continues se- 
quentially through the genome. Lastly, if a msg-handler instruction is encountered during normal sequential execution, the handler code is skipped and execution jumps past the next end-handler instruction.

In this work an interrupt handler cannot be preempted; therefore, all interrupts are handled atomically. Specifically, if a message is received while an organism is interrupted, the message is queued until the handler has finished, at which time the handler is re-entered and the next message in the queue is processed. The original context is restored only when all received messages have been processed. Furthermore, the incoming message buffer is limited to 20 messages. Messages received when the buffer is full are dropped. As we shall see in Section 3, the evolutionary process exploited this property in order to produce quorum sensing behavior. If no end-handler instruction exists then the jump is not taken.

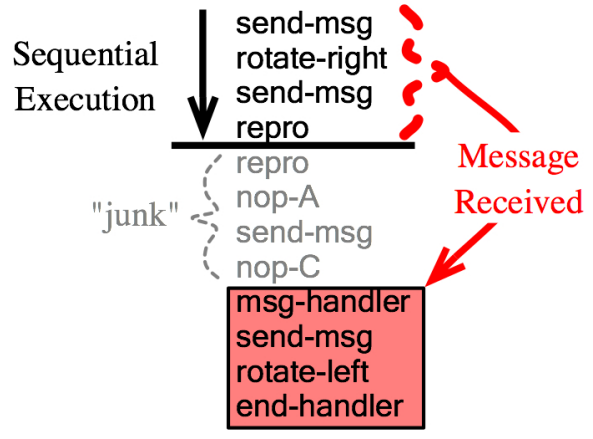

Figure 3: Sample genome containing a single interrupt handler. During sequential execution the first four instruction of this genome are executed. If a message is received the organism's IP is jumped into the interrupt handler. This example also contains "junk" code that will not be executed unless the genome is mutated.

\section{EVOLVING QUORUM SENSING}

In this section we demonstrate that the digital evolution system as described above is capable of producing deme-level populations that exhibit QS. Specifically, we observed Avida populations that evolved a group communication behavior to inhibit self-replication once a deme has reached a density threshold. Moreover, the threshold itself was not specified $a$ priori, but rather was an evolved characteristic of the population. In addition, we will show that this behavior arises under different initial conditions and is scalable to demes up to 400 times larger than the demes in which the behavior evolved.

\subsection{Experimental Setup}

In the experiments described below, multiple populations are divided into 400 demes. Each deme's topology is a $5 \times 5$ torus that is seeded with a single organism at the beginning of each competition period. A competition period lasts for 20 updates, where the average organism in the entire population will probabilistically execute 50 instructions per update. After each competition period, each deme's fitness is evaluated using Equation 3, and individual deme germlines are selected, mutated, and used to seed demes in the next competition period. Each seed organism is placed and rotated randomly, so that its initial position and facing cannot be "learned" through the evolutionary process. In addition, deme-level populations are well-mixed, meaning that during replication, the offspring is placed in a random cell within a deme, with a preference for empty cells.

This study focuses on the evolution of a digital organism's "gene" (instruction) regulation mechanism, specifically the change in an organism's instruction expression under low and high density conditions. To facilitate genome analysis, given in Section 3.4, we use an instruction set that is includes the send-msg, msg-handler, end-handler, and various rotate instructions described below. Additionally, four different no-operation instructions and the repro instruction are provided. This instruction set contains fewer instructions than previous Avida studies [15]. Other instructions could be included in the set of instructions available for mutation. However, since we are not applying any additional selective pressures, organism behaviors will evolve in accordance to the deme-level fitness function.

Treatments. Our initial experiments are divided into three treatments based on available rotation methods. There are three types: Single step, Labeled, and Neighbor-based. Single step rotations enable an organism to rotate one cell to its left or right by executing the rotate-right or rotateleft instruction, respectively. The rotate-label instruction enables an organism to rotate to a direction specified by a sequence of subsequent nop instructions, or label. (An explanation of labels and nop-modifiable instructions can be found in [22].) Lastly, the instructions rotate-occupied-neighbor and rotate-unoccupied-neighbor perform neighbor-based rotations. These instructions will rotate an organism to a neighbor cell that is occupied or unoccupied, respectively. Each treatment consists of 20 runs with each run lasting for 2500 deme competition periods (generations). Within a given run, the evolutionary process has access to only one of the three rotations methods, simplifying intra-treatment comparisons. In addition, we compare results from all treatment, however, all comparisons are limited to those runs that exhibit the desired overall behavior, namely population control. Out of the 20 runs in each treatment, we observed 15 runs in the Single step treatment and 14 runs in both the Label and Neighbor treatments that exhibited this behavior, only those runs are used in the following discussion.

\subsection{Energy Conservation}

We begin by considering the amount of energy conserved per deme for the three different treatments. This value alone determines the fitness of the deme. We note that there are only two ways for a deme to lose energy: the deme's constituents use energy when executing instructions, and the energy remaining in an organism is purged when the organism is replaced. Since every instruction has the same energy cost, different instruction execution sequences of the same length all have the same explicit energy cost. Therefore, the only way organisms in a deme can reduce their total energy usage is to limit self-replication.

Figure 4 displays the average fraction of energy remaining within a deme at the end of a competition period. Since Figure 4 shows an increase in energy conserved per deme in all treatments, it can be assumed that the number of births per deme is declining over evolutionary time. This assumption is confirmed by Figure 5, which shows the average number of births per deme. For all three treatments, the number declines to approximately 30-35 births per deme. 


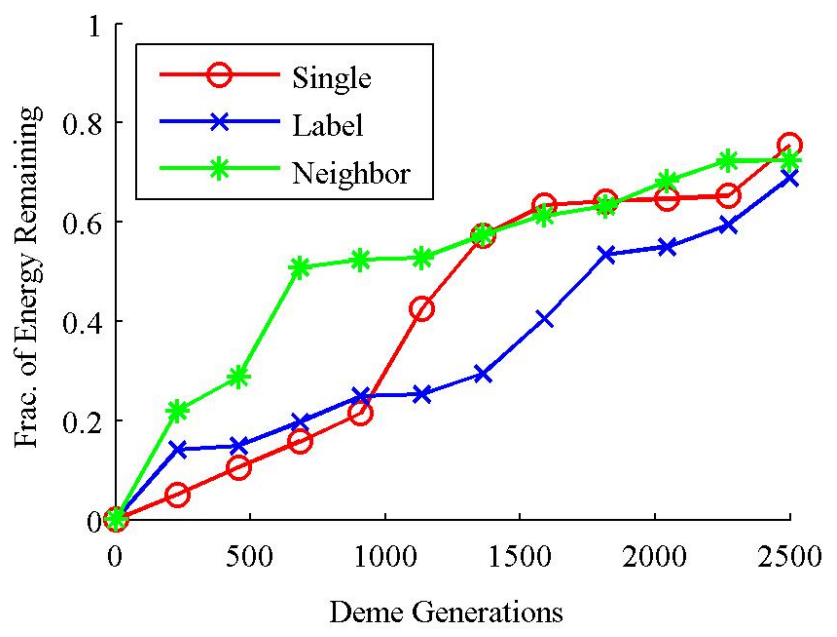

Figure 4: Mean fraction of energy remaining per deme over deme generations for each treatment.

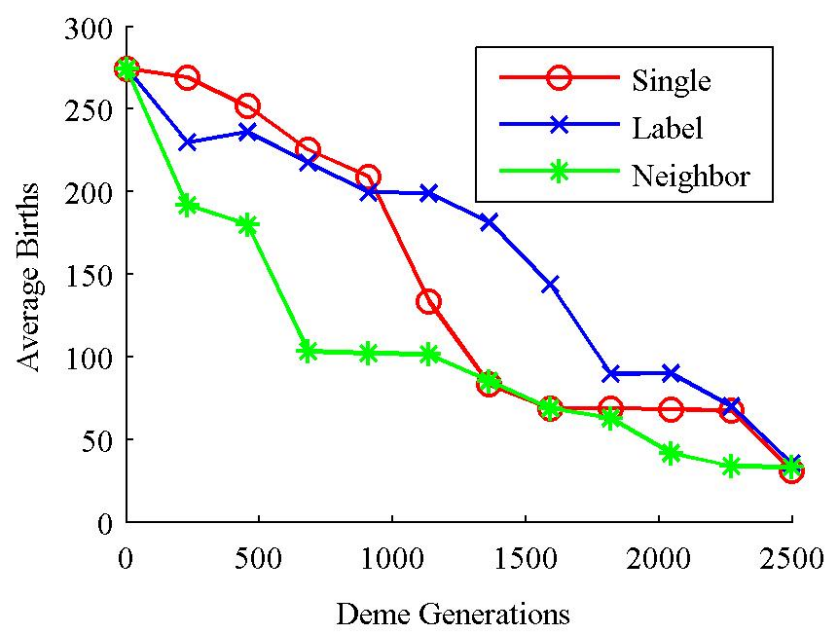

Figure 5: Average number of births per deme for all three treatments.

\subsection{Group Behavior}

QS behaviors found in natural organisms exhibit two key features. First, a density based change in behavior can be observed, and secondly, after a threshold has been reached a positive feedback loop is created that causes more AI to be released. In this paper messages are analogous to AIs, and alternate gene (instruction) activation can be realized through the evolution of an interrupt handler. We define organism density as the number of organisms per cell; therefore, a density of 1.0 can be achieved only if every cell in the environment contains an organism. Figure 6 plots the average organism density per deme over evolutionary time. Both the Label and Neighbor rotation treatments exhibit a slight, yet steady decline in organism density. However, the Single rotation treatment displays a larger decline in organism density, reaching a value of approximately 0.9 . The Single rotation instructions provided organisms with the ability to easily send a message and then rotate one cell to the left or right. Performing this basic behavior in the Label treatment requires a much longer sequence of instructions and is therefore less likely to evolve. In addition, this basic send and rotate strategy is not possible in the Neighbor treatment without additional information about the organism's current neighborhood. In the remainder of this paper we focus only on results produced in the Single rotation treatment.

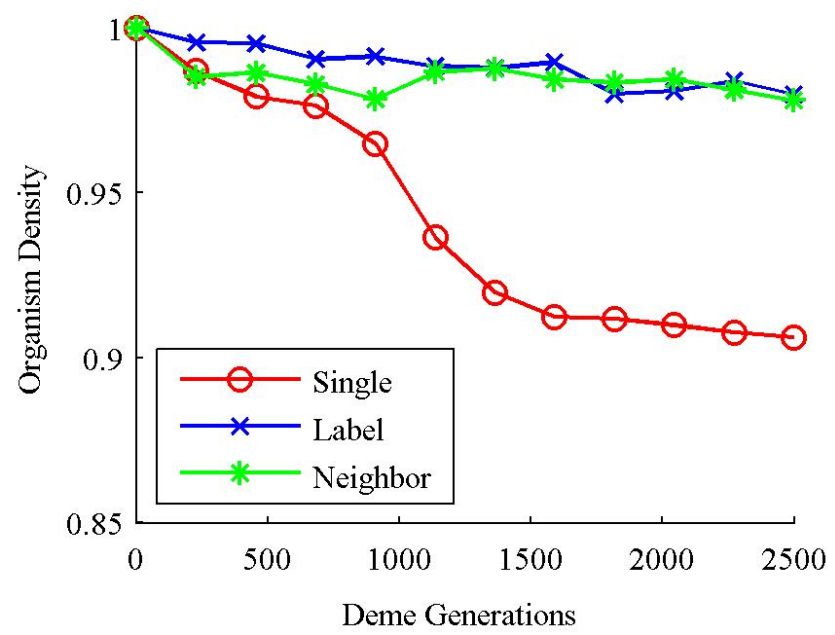

Figure 6: Average organism density per deme.

We focus on the most abundant, or dominant, genomes produced by runs. These dominant genomes are extracted from each run at its conclusion, and a replicate of each is used to seed 400 demes which are then run for one competition period. While these demes are executing, we record for each deme the organism density, total births, and number of organisms running in an interrupted state. Figure 7 shows graphically the correlation between organism density and total births per deme averaged over all dominant genomes. As seen in Figure 7, after update 4, both the organism density and total births per deme plateau. Therefore, deme-wide self-replication behavior, which is present before update 4, is suppressed when the organism density reaches approximately 0.8 , demonstrating a quorum has been reached.

To this point we have not discussed methods by which the organisms implement quorum sensing. Figure 8 plots the average number of organisms per deme that are executing in an interrupt handler. This curve closely mirrors the curves in Figure 7, providing an indication that interrupts are being used to suppress organism self-replication. In addition, if we disable messaging, therefore organisms cannot become interrupted, the average organism density within a deme quickly increase to 1.0 and all demes die out due to energy depletion. This provides further evidence that interruptcausing messages are an important feature of the evolved genomes. Now let us focus on the genome of an organism that realizes this behavior.

\subsection{Genome Analysis}

Here, we focus on the dominant genome that produced the lowest average organism density of 0.67 . An organism containing this dominant genome, shown in Figure 9, will execute the first 16 instructions in the genome before it replicates, as denoted by the black line to the left of the genome. During this sequence the organism sends a single message 


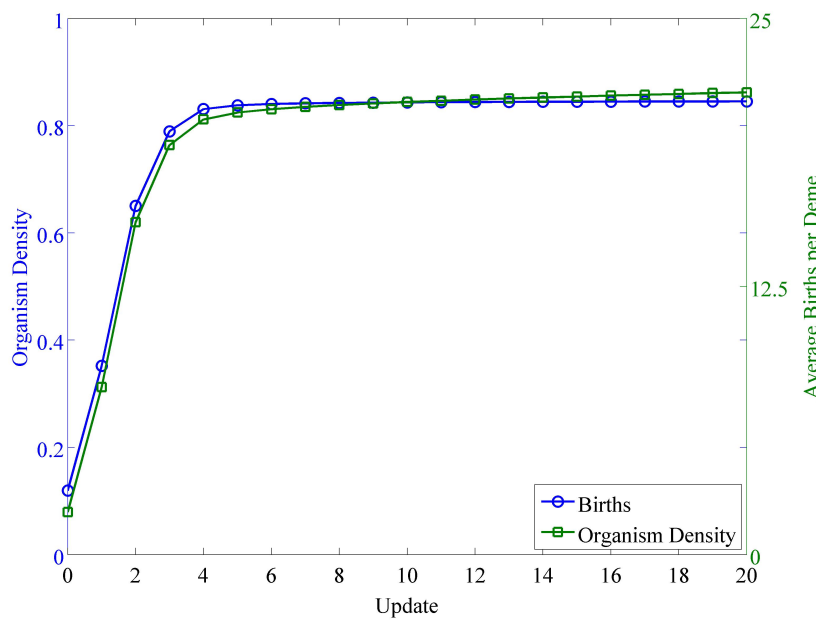

Figure 7: Organism density and total births per deme for dominate organisms.

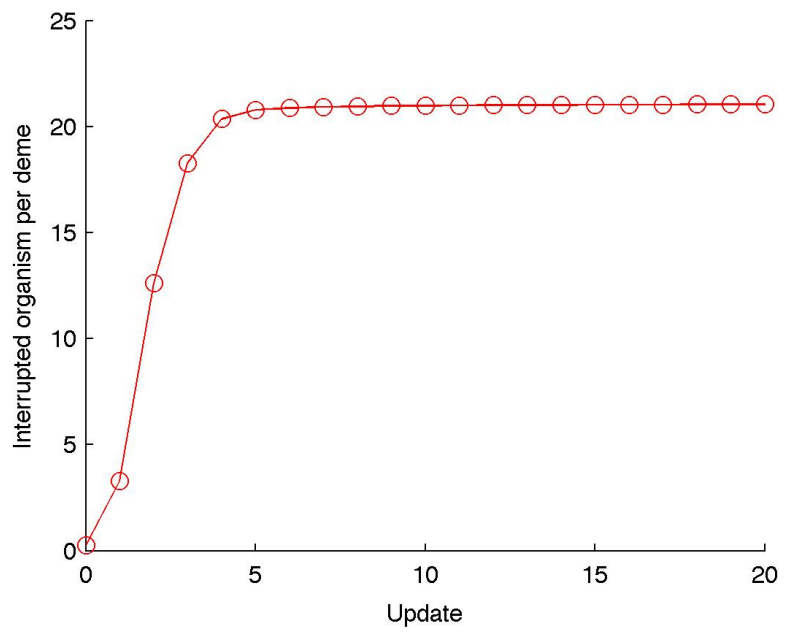

Figure 8: Average number of organisms interrupted per deme.

to its initially faced cell and the two neighboring cells to its left, finally replicating while facing the cell one rotation to the left of its initial facing. In short, the organism will send 3 messages for every 16 executed instructions during normal execution and then replicate. Upon replication the organism's state is reset, causing the genome to be processed from the beginning. Since genomes are executed in a cyclic manner, this sequence will be repeated until the organism either runs out of energy, is replaced by an offspring of another organism, or is interrupted. If interrupted, the current context of this organism is saved and the interrupt-causing message is processed in the interrupt handler, denoted by the red boxes at the bottom (beginning of handler) and top (end of handler) of the genome in Figure 9. While interrupted, the organism will send one message to the cell it currently faces and two messages to the cell left of its initial facing. Moreover, the organism will remain in the interrupt handler, and hence will not replicate, until all received messages have been processed. Furthermore, for every entrance into the interrupt handler caused by receiving a message, the organism will produce three messages. Hence execution of the interrupt handler produces a positive feedback loop where the level of messaging (AI) in the system is increased, thus tripling the chances than an organism will become interrupted. Therefore, the expression of this individual behavior in a dense group of digital organisms will cause an organism to remain in a state of perpetual interruption and never self-replicate.

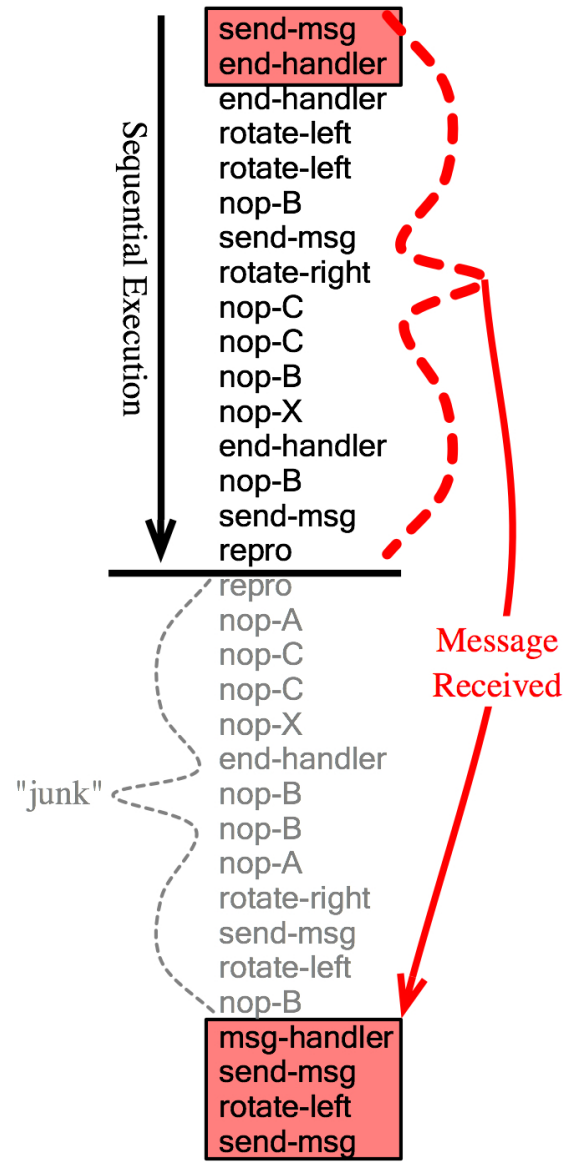

Figure 9: Evolved dominate genome that produces the lowest average organism density.

\subsection{Experiments with Larger Demes}

To determine whether this QS behavior is truly density based we seeded demes several times larger than the original $5 \times 5$ demes in which the behavior evolved. Table 1 provides a list of deme sizes tested, their scale relative to a $5 \times 5$ deme, total number of demes per run, and total number of demes used to generate averages plotted in Figure 10. Note that only 15 of the 20 runs evolved population control behavior, so only those dominants were used, producing the values in column 4 of Table 1. Each deme is again allowed to execute for a single competition period and the average organism density for all three scalings over time is shown in Figure 10. The final average densities produced for all three scalings are insignificantly different. Therefore, the evolved dominant genomes exhibit a quorum sensing behavior that suppresses organism self-replication under multiple scalings. In addition, a quorum is reached at similar organism densities. 
Table 1: Deme size comparision

\begin{tabular}{|l|c|c|c|}
\hline deme size & $N \times$ larger & \# demes & total demes \\
\hline \hline $5 \times 5$ & 1 & 400 & 6000 \\
\hline $25 \times 25$ & 25 & 20 & 300 \\
\hline $50 \times 50$ & 100 & 10 & 150 \\
\hline
\end{tabular}

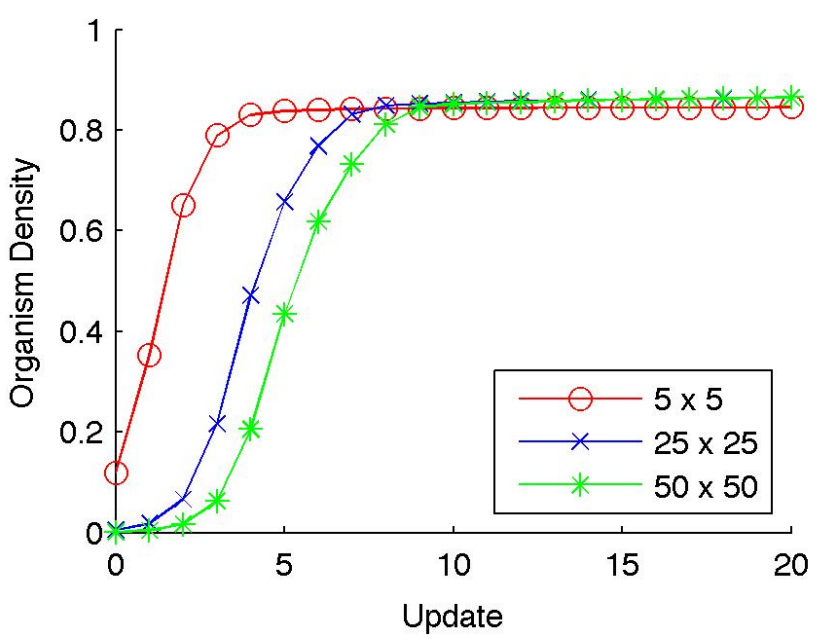

Figure 10: Average organism density under multiple deme sizes.

Finally, we seeded a single $100 \times 100$ deme with an organism containing the genome in Figure 9. We then allowed the deme to execute for one competition period, and we tracked the constituent organisms' execution behavior. Figure 11 shows snapshots of the resulting behavior, where each $(x, y)$ coordinate corresponds to a single cell in the $100 \times 100$ deme. A cell is colored according to the current activity within that cell. If a cell does not contain an organism, it is colored white. A cell that contains an uninterrupted organism is colored black, and a red cell denotes the presence of an organism in an interrupted state. As depicted in Figure 11, the population quickly switches behaviors when it becomes dense. In fact, the time difference between Figures 11(c) and 11(e) is less than two updates. The rapid change is behavior is a hallmark of QS and has been observed in natural and now digital organisms.

\section{CONCLUSIONS}

In this paper we have demonstrated the evolution of quorum sensing behavior in digital organisms. Specifically, we examined the evolution of behavior that suppresses individual self-replication under high density conditions. First, we showed that this type of behavior evolves in a majority of runs in all three of our rotation treatments. Second, we extracted the dominant genomes from the single rotation treatments and showed the tight correlation between organism density, total births, and number of interrupted organisms. Third, we discussed the individual behavior of the dominant genome that produced the lowest organism density. Fourth, we increased the size of the demes by two orders of magnitude and showed the average organism density remained the same. Lastly, we visualized the rapid change in behavior of a dominant organism in a deme 400 times larger than the environment it evolved in.
Continuing with this line of research, we intend to extend the interrupt model to incorporate active messages, enabling the sending of a message to a specific handler. This extension will move us one step closer to the TinyOS execution model, enabling us to evolve digital organisms with capabilities similar to those of wireless sensor network nodes. We also intend to add individual tasks in order to study tradeoffs between individual and group-level behaviors. Lastly, we plan to remove the intra-deme mutation restriction that we used in this paper, so that our model will more closely resemble the natural world.

Acknowledgments. This work was supported by NSF Grants CCF-0750787, CNS-0751155, CCF-0820220, by U.S. Army Grant W911NF-08-1-0495, and by a Quality Fund Grant from Michigan State University.

\section{REFERENCES}

[1] C. Adami, C. A. Ofria, and T. C. Collier. Evolution of biological complexity. Proceedings of the National Academy of Sciences, 97(9):4463-4468, April 2000.

[2] O. Babaoglu, G. Canright, A. Deutsch, G. A. D. Caro, F. Ducatelle, L. M. Gambardella, N. Ganguly, M. Jelasity, R. Montemanni, A. Montresor, and T. Urnes. Design patterns from biology for distributed computing. ACM Transactions on Autonomous and Adaptive Systems, 1(1):26-66, 2006.

[3] O. Béjà, E. N. Spudich, J. L. Spudich, M. Leclerc, and E. F. DeLong. Proteorhodopsin phototrophy in the ocean. Nature, 411:786-789, 2001.

[4] R. D. Berg. The indigenous gastrointestinal microflora. Trends in Microbiology, 4(11):430 - 435, 1996.

[5] F. Bernardini, M. Gheorghe, and N. Krasnogor. Quorum sensing P systems. Theor. Comput. Sci., 371(1-2):20-33, 2007.

[6] S. P. Brown and R. A. Johnstone. Cooperation in the dark: Signalling and collective action in quorum-sensing bacteria. Proc. of the Royal Society of London B: Biological Sciences, 268(1470):961-965, 2001.

[7] B. Crespi. The evolution of social behavior in microorganisms. Trends in Ecology and Evolution, 16(4), 2001.

[8] D. Davies, M. Parsek, J. Pearson, B. Iglewski, J. Costerton, and E. Greenberg. The involvement of cell-to-cell signals in the development of a bacterial biofilm. Science, 280(5361):295-8, Apr. 1998.

[9] T. R. de Kievit and I. B. H. Bacterial quorum sensing in pathogenic relationships. Infect. Immun., 68(9):4839-4849, September 2000.

[10] M. Dworkin and D. Kaiser. Cell interactions in myxobacterial growth and development. Science, 230(4721):18-24, Oct. 1985.

[11] D. Floreano, S. Mitri, S. Magnenat, and L. Keller. Evolutionary conditions for the emergence of communication in robots. Current Biology, 17:514-519, March 2007.

[12] S. K. Hansen, P. B. Rainey, J. A. J. Haagensen, and S. Molin. Evolution of species interactions in a biofilm community. Nature, 445:533-536, February 2007.

[13] J. Hill, R. Szewczyk, A. Woo, S. Hollar, D. Culler, and K. Pister. System architecture directions for networked sensors. SIGPLAN Not., 35(11):93-104, 2000. 


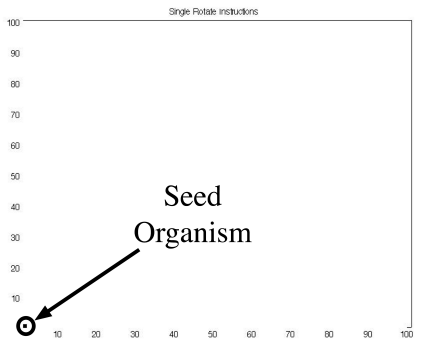

(a) $\mathrm{t}=0.0$ updates

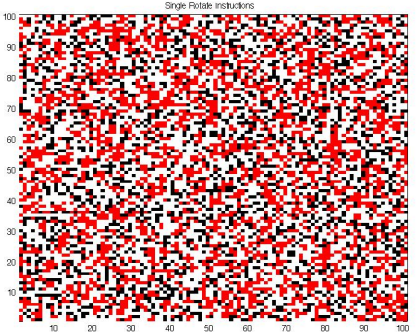

(d) $\mathrm{t}=4.8$ updates

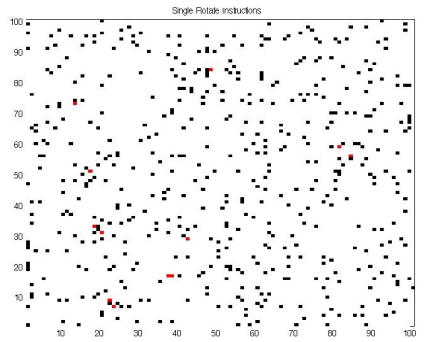

(b) $\mathrm{t}=3.2$ updates

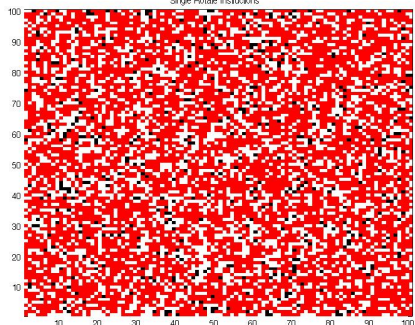

(e) $\mathrm{t}=5.6$ updates

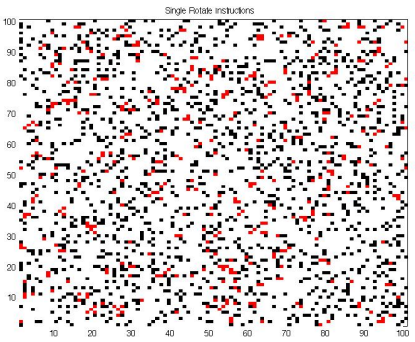

(c) $\mathrm{t}=4.0$ updates

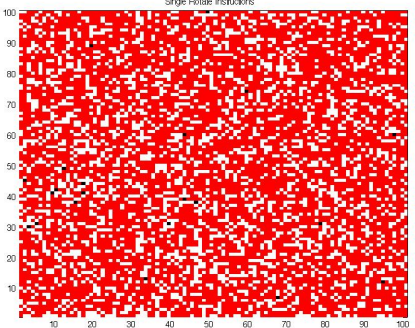

(f) $\mathrm{t}=8.0$ updates

Figure 11: Images of a $100 \times 100$ deme initially seeded with the genome shown in Figure 9. Figure 11(a) shows the initial state of the deme with a single uninterrupted organism in the lowest, left-most cell. Figures 11(b) and 11(c) show a steady exponential increate in population size where the majority of organisms are executing sequentially. Figures $11(\mathrm{~d})$ and $11(\mathrm{e})$ depict the rapid behavioral change from self-replication to suppression of self-replication. Lastly, Figure 11(f) shows the state of the deme population $40 \%$ of the way through a competition period.

[14] R. E. Lenki, C. A. Ofria, T. C. Collier, and C. Adami. Genome complexity, robustness and genetic interactions in digital organisms. Nature, 400:661-664, 1999.

[15] R. E. Lenski, C. Ofria, R. T. Pennock, and C. Adami. The evolutionary origin of complex features. Nature, 423:139-144, 2003.

[16] R. C. Massey, M. J. Horsburgh, G. Lina, M. Höök, , and M. Recker. The evolution and maintenance of virulence in staphylococcus aureus: a role for host-to-host transmission? Nature Reviews Microbiology, 4:953-958, December 2006.

[17] P. McKinley, B. Cheng, C. Ofria, D. Knoester, B. Beckmann, and H. Goldsby. Harnessing digital evolution. IEEE Computer, 41(1):54-63, January 2008.

[18] M. B. Miller and B. L. Bassler. Quorum sensing in bacteria. Annu. Rev. Microbiol, 55:165-199, 2001.

[19] C. D. Nadell, J. B. Xavier, S. A. Levin, and K. R. Foster. The evolution of quorum sensing in bacterial biofilms. PLoS Biology, 6(1):0171-0179, January 2008.

[20] K. Nealson and J. Hastings. Bacterial bioluminescence: its control and ecological significance. Microbiol. Rev., 43(4):496-518, 1979.

[21] K. H. Nealson, T. Platt, and J. W. Hastings. Cellular control of the synthesis and activity of the bacterial luminescent system. J Bacteriol., 104(1):313-322, 1970.

[22] C. Ofria and C. O. Wilke. Avida: A software platform for research in computational evolutionary biology. Artificial Life, 10:191-229, March 2004.
[23] J. Park, R. Jagasia, G. F. Kaufmann, J. C. Mathison, D. I. Ruiz, J. A. Moss, M. M. Meijler, R. J. Ulevitch, and K. D. Janda. Infection control by antibody disruption of bacterial quorum sensing signaling. Chemistry \& Biology, 14(10):1119-1127, Oct. 2007.

[24] J. R. Porter. Antony van leeuwenhoek: tercentenary of his discovery of bacteria. Microbiol. Mol. Biol. Rev., 40(2):260-269, 1976.

[25] F. J. Romero-Campero and M. J. Pérez-Jiménez. A model of the quorum sensing system in Vibrio fischeri using P systems. Artificial Life, 14(1):95-109, 2008.

[26] G. Terrazas, N. Krasnogor, M. Gheorghe, F. Bernardini, S. Diggle, and M. Cámara. An environment aware P-System model of quorum sensing. In S. B. Cooper, B. Löwe, and L. Torenvliet, editors, New Computational Paradigms, volume 3526 of Lecture Notes in Computer Science, pages 479-485. Springer, 2005.

[27] C. M. Waters and B. L. Bassler. Quorum sensing: Cell-to-cell communication in bacteria. Annual Review of Cell and Developmental Biology, 21:319-346, 2005.

[28] D. Whitley, S. Rana, and R. B. Heckendorn. Island model genetic algorithms and linearly separable problems. In Proceedings of AISB Workshop on Evolutionary Computation, volume 1305 of LNCS, pages 109-125. Springer-Verlag, 1997.

[29] D. S. Wilson. Introduction: Multilevel selection theory comes of age. The American Naturalist, 150(S1-S4), July 1997. 